\title{
A Brief Study on a Novel Approach for the Gearbox Fault Diagnosis
}

\author{
$1^{\text {st }}$ Subrata Mukherjee \\ Department of Mechanical Engineering \\ Thapar Institute of Engineering and Technology \\ Patiala, Punjab 147004, India \\ subrata.re.me@gmail.com
}

\author{
$2^{\text {nd }}$ Vikash Kumar \\ Department of Mechanical Engineering \\ Indian Institute of Technology Patna \\ Bihta, Bihar 801106, India \\ 1821me18@iitp.ac.in
}

\author{
$3^{\text {rd }}$ Somnath Sarangi \\ Department of Mechanical Engineering \\ Indian Institute of Technology Patna \\ Bihta, Bihar 801106, India \\ somsara@ iitp.ac.in
}

\begin{abstract}
Fault diagnosis of the gearbox is a decisive part of the modern industry to find the many gearbox defects like gear tooth crack, chipped or broken etc. But sometimes, the nonstationary properties of vibration signal and low energy of minimal faults makes this procedure very challenging. Previously, many types of techniques have been developed for gearbox condition monitoring. But most of the methods are dealing with conventional techniques of the gearbox condition monitoring, such as time-domain analysis or frequency domain analysis. Most of the conventional methods are not suitable for the nonstationary vibration signal. Thus, this paper presents a novel gearbox fault diagnosis technique using conditional temporal moments and optimizable support vector machine (SVM). This work also presents an integrated features extraction technique based on the standard features, i.e., statistical and spectral features with the combinations of moments features. The impact of the four conditional temporal moments of each gearbox conditions is also presented. This work shows that the proposed method successfully classifies and categorizes the gearbox faults at an early stage.
\end{abstract}

Index Terms-Conditional temporal moments, Optimizable support vector machine (SVM), Gearbox fault diagnosis, Vibration analysis.

\section{INTRODUCTION}

Gearbox is one of the most crucial elements of any machinery, such as the automotive, turbine [1]-[3]. Due to extreme load and colossal friction, the gearbox often displays some irregular behaviour known as a gearbox failure [1], [2]. The gearbox's failure occurs mainly due to an internal fault related to the gear tooth's deterioration or damage [1], [2]. The gearbox's vibration study is a meaningful way to detect faults, and the constant observation of the gearbox vibration signals detects the exact gearbox failure, which said the apparatus' imperfection to schedule the best possible to maintain a strategic gap from the surprising shutdown [1], [2].

Various sophisticated signal processing approaches can successfully evaluate the vibration signal with or without nonlinearity to diagnose the gearbox faults [4]-[6]. Several features need to be determined from the vibration signal to consider the fault characteristics' behaviour [7]-[9]. Numerous machine learning techniques are presently used to extract, select, and classify the gearbox's vibration features [7]-[9]. Currently, the support vector machine (SVM) is a commonly used AI technique for gearbox fault diagnosis [10], [11].

An evident and correct knowledge of the vibration signal about its impacts is vital for the smooth diagnosis of the gearbox faults. Countless time domain and frequency domain signal preprocessing methods can remove the non-linear effects, i.e., noise, from the vibration signal such as TSA [12], [13], energy operator [2], [14]. Also, various typical frequency domain assessments are available to analyze the gearbox faults, but these cannot determine the vibration response's time-varying frequency behaviour [4].

Time-frequency moments provide an economical way to describe the vibration response's nonstationary and timevarying frequency behaviour [15]-[20]. The nonstationary response occurs due to the rotating machines with degraded internal or external components [1]-[8]. Also, some authors investigated hyperparameter optimization in classifications [21]-[31]. Correspondingly, automated machine learning was used in hyperparameter optimizations [21]-[31].

Therefore, this paper attempts to study a novel fault diagnosis technique based on the conditional temporal moments and optimizable support vector machine. This paper also introduces four conditional time-frequency distribution for each gearbox state's vibration response, i.e., healthy, chipped, and a missing tooth at the speed of $15 \mathrm{~Hz}$ and loading condition of 0lb. This work deals with the extraction of standard and moments features from the gearbox's vibration response. 
The paper's remaining part is presented in this way: in section II, the experimental setup/data set. Section III, results and discussion and Section IV, the conclusion of this research work.

\section{EXPERIMENTAL INVESTIGATIONS}

Experimental work was conducted by the MFS, as shown in Fig. 1. The complete experimental configuration consists of four main subsystems: MFS, Oros NV gate data acquisition system, various sensors, and a computer.

Fig. 2 indicates the gears' faulty conditions used in the experimental study as a missing tooth and chipped tooth gearbox. Fig. 3 shows the vibration response of each gearbox condition. Experimental work was completed with a load of Olb and a speed of $15 \mathrm{~Hz}$ for the healthy, chipped and missing tooth conditions.

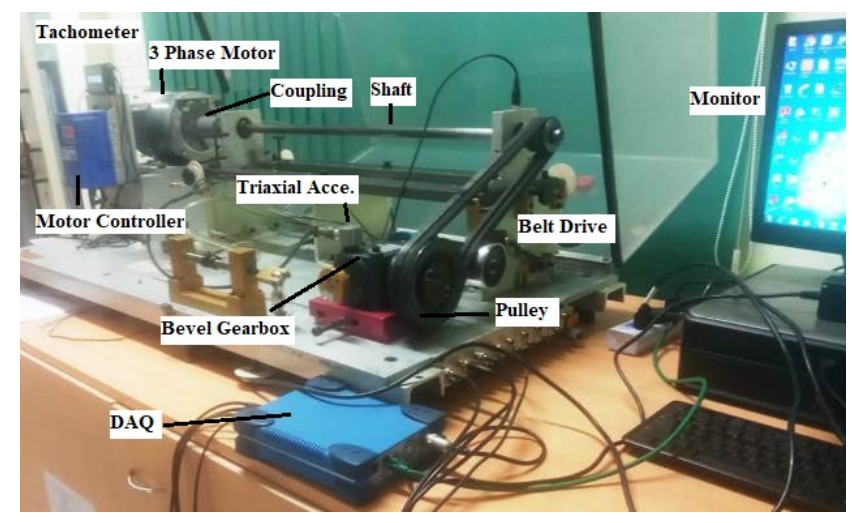

Fig. 1. Experimental setup.

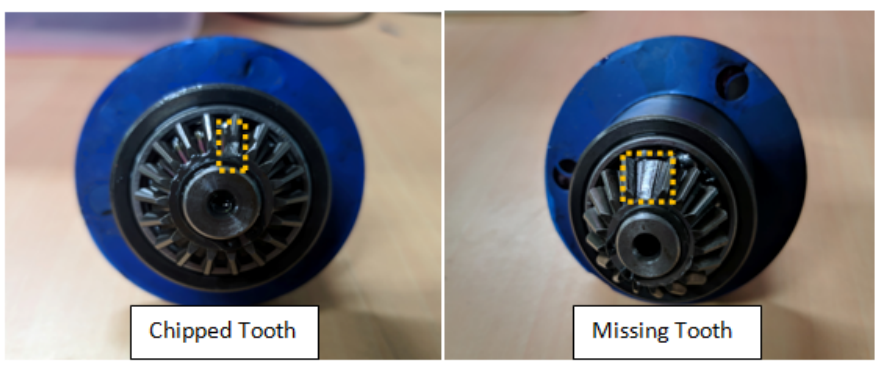

Fig. 2. Faulty gear sets.

\section{RESULTS AND DISCUSSIONS}

If we assume a density function $\rho(x)$ then from the first order to forth order [16]-[19]:

$$
\begin{gathered}
\text { Mean }, u=\int x \rho(x) d x \\
\text { Variance, } \delta^{2}=\int(x-u)^{2} \rho(x) d x \\
\text { Skew, } U=1 / \delta^{3} \int(x-u)^{3} \rho(x) d x
\end{gathered}
$$
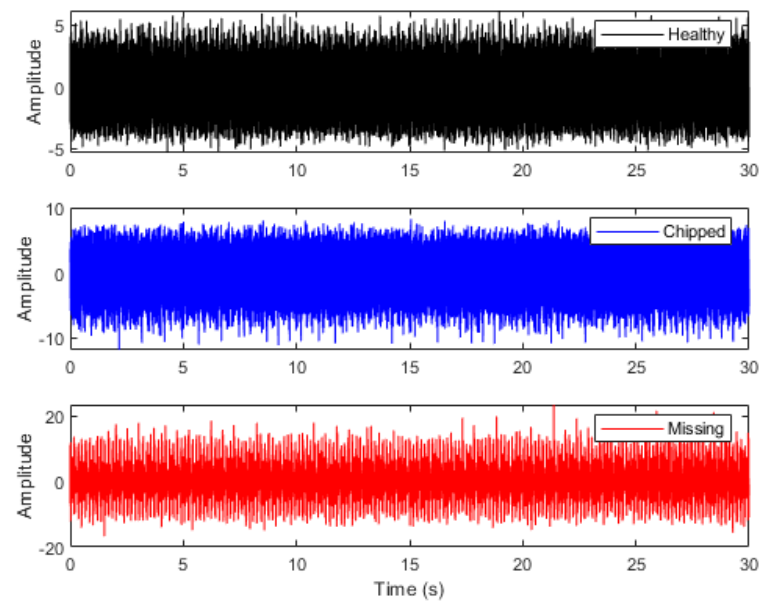

Fig. 3. Vibration response at different fault condition.

$$
\text { Kurtosis, } K=1 / \delta^{4} \int(x-u)^{4} \rho(x) d x
$$

In the Equations 5-8, the define the conditional moments in terms of $\rho(x, y)[16]-[19]$ :

Mean for a given $y$

$$
u_{x \mid y}=\int x \rho(x \mid y) d x
$$

Variance for a given $\mathrm{y}$

$$
\delta_{x \mid y}^{2}=\int\left(x-u_{x \mid y}\right)^{2} \rho(x \mid y) d x
$$

Skew for a given $y$

$$
U_{x \mid y}=1 /\left(\delta_{x \mid y}^{3}\right) \int\left(x-u_{x \mid y}\right)^{3} \rho(x \mid y) d x
$$

Kurtosis for a given $\mathrm{y}$

$$
K_{x \mid y}=1 /\left(\delta_{x \mid y}^{4}\right) \int\left(x-u_{x \mid y}\right)^{4} \rho(x \mid y) d x
$$

Where, $\rho(x \mid y)$ known as the conditional density of $\mathbf{x}$, related to joint density using Bayes formulations [16]-[19]. Figure 4-6, shows the representations of four conditional temporal moments of the healthy, chipped and missing tooth gearbox. Conditional temporal moments catch the changes in timevarying frequency behaviour of all three conditions of the gearbox. Findings suggest that these conditional temporal moments effectively used in the vibration response, which have a simple physical meaning, are useful for tracking, diagnosing, and even automatic classification for gearbox faults' continuous propagation.

Fig. 7 indicates the conventional fault diagnostic procedure. However, some improvements are needed in the conventional procedure to support a smooth diagnosis of gearbox faults due to several limitations. Fig. 8 shows the proposed schema of the fault diagnostic procedure. In this work, moment features have been incorporated with the vibration data's statistical and 

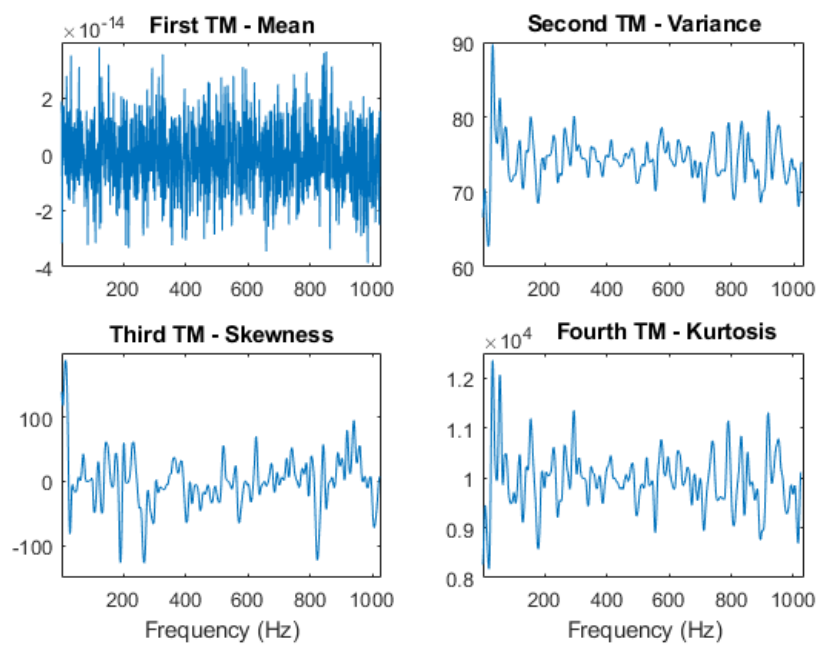

Fig. 4. Conditional temporal moments of healthy tooth gearbox.
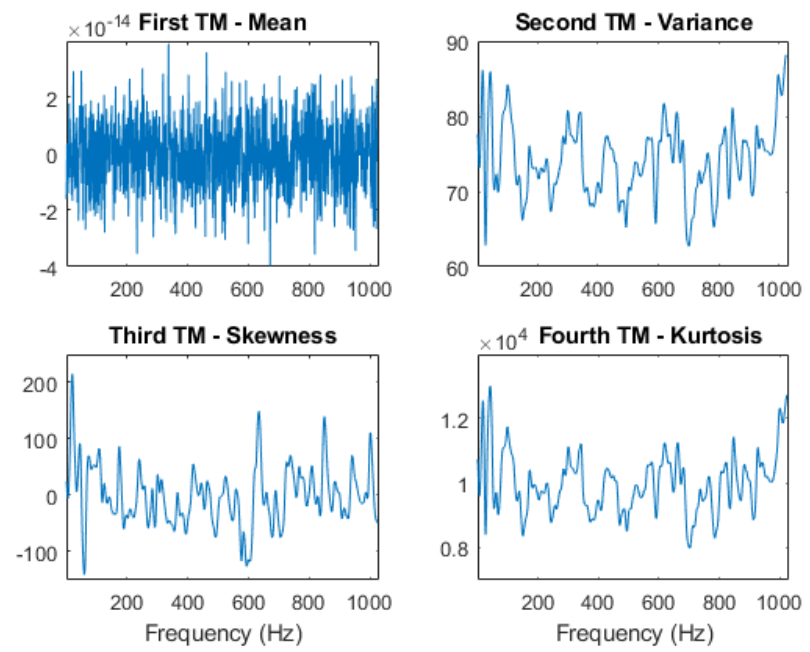

Fig. 5. Conditional temporal moments of chipped tooth gearbox.
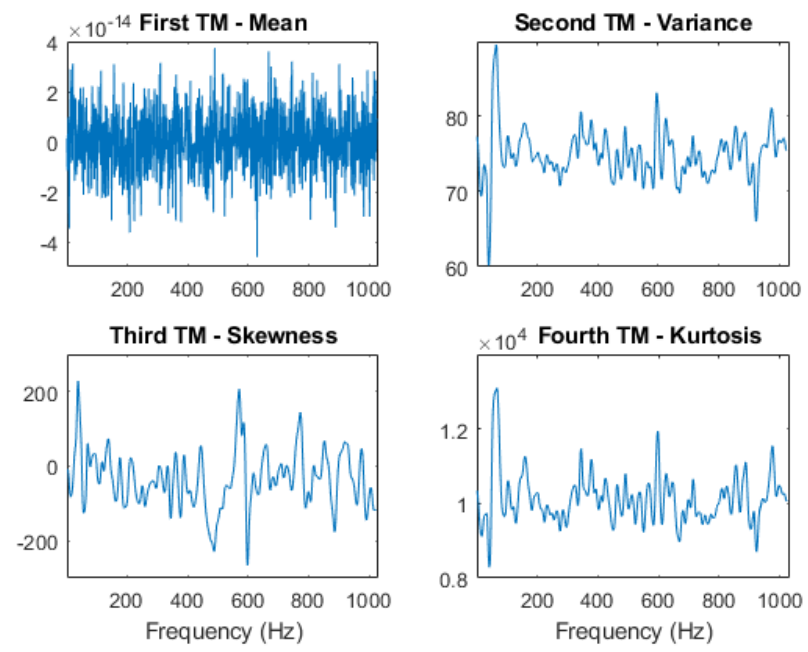

Fig. 6. Conditional temporal moments of missing tooth gearbox. spectral characteristics to diagnose the gearbox faults along with a precise time-frequency distribution, presented in Fig. 9 .

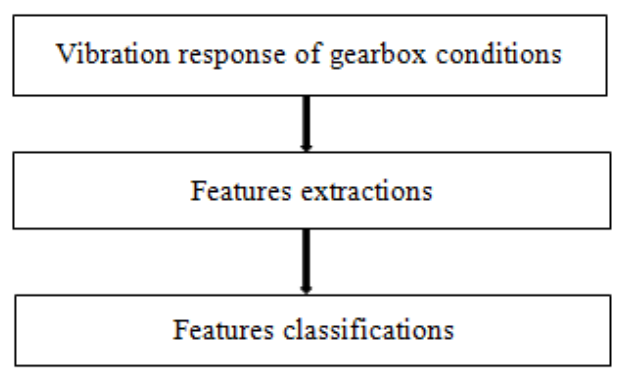

Fig. 7. Conventional fault diagnosis technique.

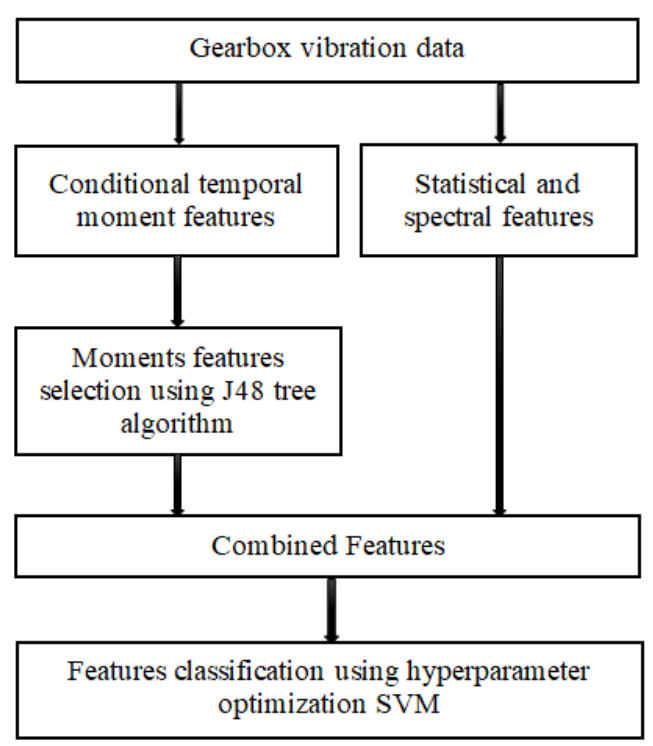

Fig. 8. Proposed fault diagnosis technique.

In recent years, the research studies on complex and computationally intensive multi-hyperparameter machine learning models, such as Automated Machine-Learning (AutoML), has resulted in a resurgence of hyperparameter optimization research (HPO) [15]-[20].

Automated hyperparameter optimization (HPO) has several critical use cases, such as (a) HPO can reduce the human work necessary to implement machine learning. In the sense of $\mathrm{Au}-$ toML, this is especially relevant (b) HPO boost the efficiency of machine learning algorithms and (c) HPO can strengthen the reproducibility and integrity of scientific research [15]-[20].

Optimizable SVM was used to classify the momentstraditional hybrid features. Four conditional temporal moment features have been determined. The meaningful moment features, i.e., second moments-variance and third momentsskewness, have been chosen using the J48 tree algorithm, presented in Fig. 10 as an input parameter in Optimizable SVM with conventional features. 


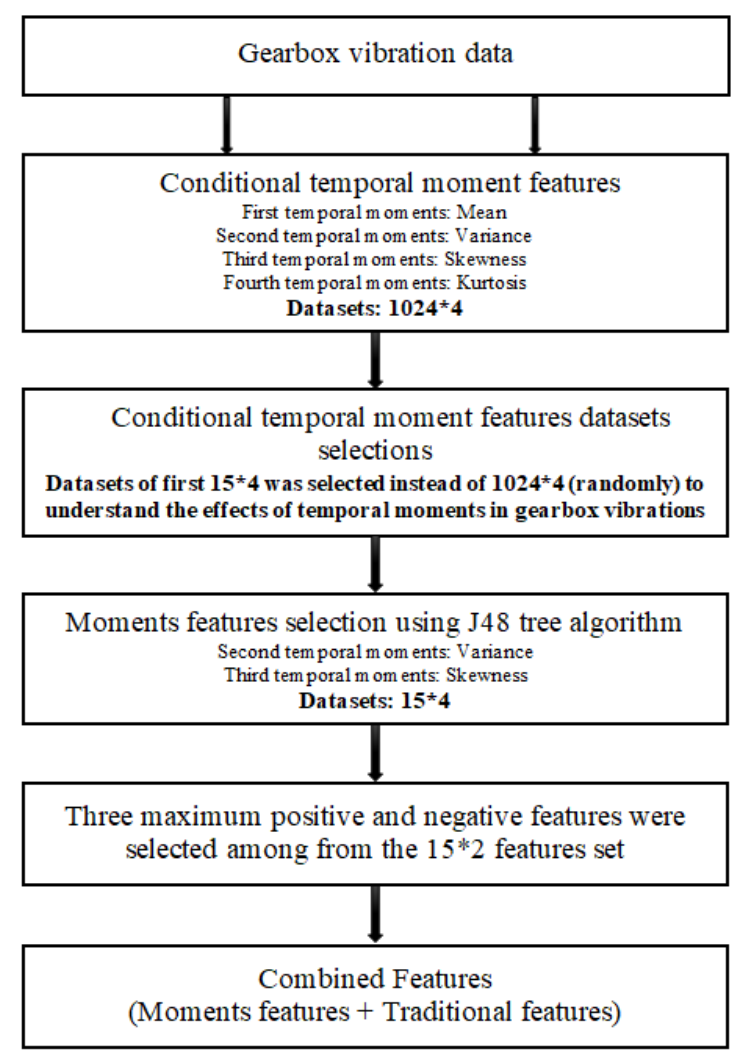

Fig. 9. Proposed features extractions technique.

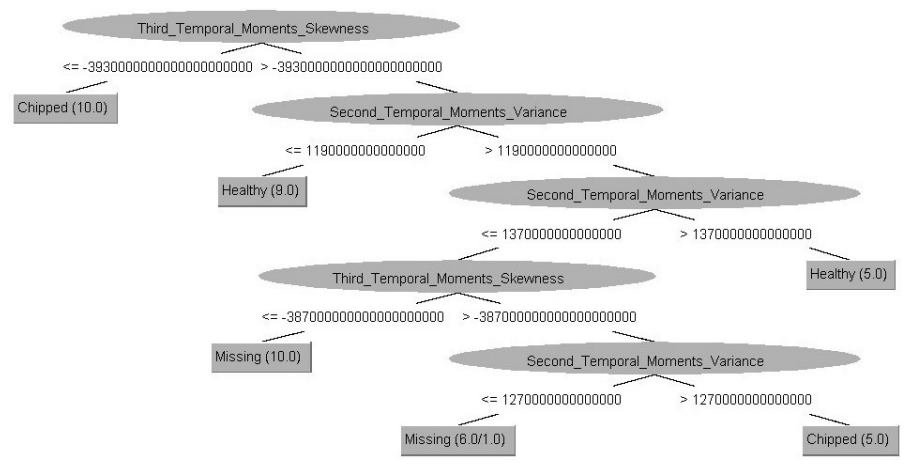

Fig. 10. Temporal moment features selection using J48 algorithm [13].

Fig. 11 signifies the several observations in each cell which was a hyperparameter-based advanced SVM classifier. Here, each cell indicates the three significant cases of features with the three different gearbox conditions.

Fig. 12 displays the minimum classification error plot for the optimizable SVM. In this work, the linear kernel function was used. The four plots are showing in the graphs are (a) Estimated minimum classification error, (b) best point hyperparameters, (c) observed minimum classification error and (d) best point hyperparameters. In [32], more explanations are demonstrated about minimum classification error plot. Optimizable SVM reveals $88.9 \%$ efficiency in classification, significantly improving classification relative to the moments and conventional classification strategies.

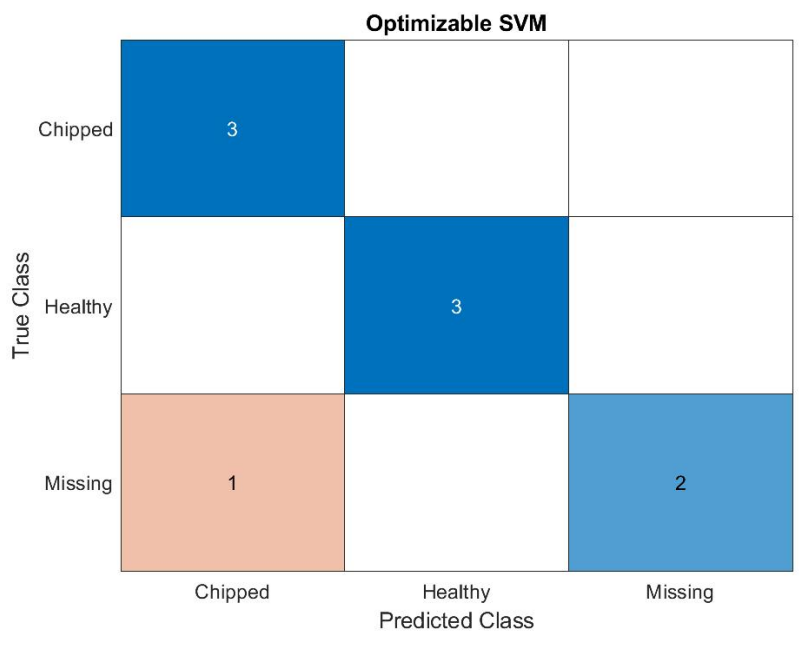

Fig. 11. Confusion matrix of combined features.

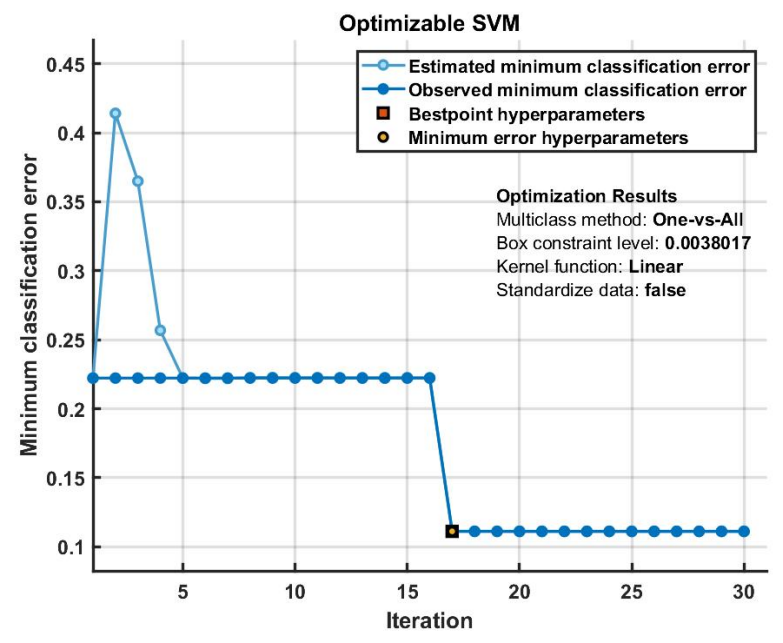

Fig. 12. Minimum classification error plot.

\section{CONCLUSION}

- This paper presents an experimental study based on the gearbox fault diagnosis using conditional temporal moments and optimizable SVM. The presented work was an experimental attempt to demonstrate the effects of gearbox vibration's conditional moments.

- This work also presented the changes in the conditional temporal moment for the gearbox's three different cases at the speed of $15 \mathrm{~Hz}$ and loading conditions of $0 \mathrm{lb}$. The effects of the four moments are observable in Figs 4-6.

- For easy understanding, only one speed and one load condition of the gearbox were considered. Authors believed that this study could be performed in the more significant number of cases or conditions of any rotating machinery for the fault's diagnosis. 
- An AutoML algorithm, hyperparameter optimizable SVM,was introduced to classify the hybrid features, i.e., conditional temporal-traditional features, which was showed $88.9 \%$ accuracy with a minimum classification error plot, presented in Figs 11-12.

- In future, this study can implement the complex gear dynamics to classify the various gear tooth damage. Also, it can be implemented on the higher-order faults in the experimental vibration data.

- Noise optimization or noise cancellations based advanced signal preprocessing techniques can also be implemented with moments features to diagnose the gearbox faults. Also, this study can be used in the hybrid classifications techniques in case of faults diagnosis.

- Classification accuracy of the four moments features presented in Table I, showing an individual confusion matrix using J48 algorithm [13] with the accuracy of 88.9 $\%$.

- Spectrograms of the gearbox vibration also presented in the Figs 13-15 at $15 \mathrm{~Hz}$ (speed) and 0lb (load)

\section{ACKNOWLEDGMENT}

This work was supported by Dynamics of Machinery lab IIT Patna.

\section{REFERENCES}

[1] Wei, Y., Li, Y., Xu, M., \& Huang, W. (2019). A review of early fault diagnosis approaches and their applications in rotating machinery. In Entropy. https://doi.org/10.3390/e21040409

[2] Sharma, V., \& Parey, A. (2016). A Review of Gear Fault Diagnosis Using Various Condition Indicators. Procedia Engineering. https://doi.org/10.1016/j.proeng.2016.05.131

[3] Feng, Z., Liang, M., Zhang, Y., \& Hou, S. (2012). Fault diagnosis for wind turbine planetary gearboxes via demodulation analysis based on ensemble empirical mode decomposition and energy separation. Renewable Energy. https://doi.org/10.1016/j.renene.2012.04.019

[4] Feng, Z., Liang, M., \& Chu, F. (2013). Recent advances in timefrequency analysis methods for machinery fault diagnosis: A review with application examples. Mechanical Systems and Signal Processing. https://doi.org/10.1016/j.ymssp.2013.01.017

[5] Rai, A., \& Upadhyay, S. H. (2016). A review on signal processing techniques utilized in the fault diagnosis of rolling element bearings. Tribology International. https://doi.org/10.1016/j.triboint.2015.12.037

[6] Li, S., Xin, Y., Li, X., Wang, J., \& Xu, K. (2019). A review on the signal processing methods of rotating machinery fault diagnosis. Proceedings of 2019 IEEE 8th Joint International Information Technology and Artificial Intelligence Conference, ITAIC 2019. https://doi.org/10.1109/ITAIC.2019.878557

[7] Lei, Y., Yang, B., Jiang, X., Jia, F., Li, N., \& Nandi, A. K. (2020). Applications of machine learning to machine fault diagnosis: A review and roadmap. In Mechanical Systems and Signal Processing. https://doi.org/10.1016/j.ymssp.2019.106587

[8] Caesarendra, W., \& Tjahjowidodo, T. (2017). A review of feature extraction methods in vibration-based condition monitoring and its application for degradation trend estimation of low-speed slew bearing. In Machines. https://doi.org/10.3390/machines5040021

[9] Djenouri, D., Laidi, R., Djenouri, Y., \& Balasingham, I. (2019). Machine learning for smart building applications: Review and taxonomy. ACM Computing Surveys. https://doi.org/10.1145/3311950

[10] Cervantes, J., Garcia-Lamont, F., Rodríguez-Mazahua, L., \& Lopez, A. (2020). A comprehensive survey on support vector machine classification: Applications, challenges and trends. Neurocomputing. https://doi.org/10.1016/j.neucom.2019.10.118

[11] Liu, R., Yang, B., Zio, E., \& Chen, X. (2018). Artificial intelligence for fault diagnosis of rotating machinery: A review. In Mechanical Systems and Signal Processing. https://doi.org/10.1016/j.ymssp.2018.02.016
[12] Bechhoefer, E., \& Kingsley, M. (2009). A review of time-synchronous average algorithms. Annual Conference of the Prognostics and Health Management Society, PHM 2009.

[13] Mukherjee, S., Kumar, V., Sarangi, S., \& Bera, T. K. (2020). Gearbox Fault Diagnosis using Advanced Computational Intelligence. Procedia Computer Science. https://doi.org/10.1016/j.procs.2020.03.370.

[14] Feng, Z., Zuo, M. J., Hao, R., Chu, F., \& Lee, J. (2013). Ensemble empirical mode decomposition-based teager energy spectrum for bearing fault diagnosis. Journal of Vibration and Acoustics, Transactions of the ASME. https://doi.org/10.1115/1.4023814

[15] Yesilyurt, I. (2004). The application of the conditional moments analysis to gearbox fault detection - A comparative study using the spectrogram and scalogram. NDT and E International, 37(4), 309-320. https://doi.org/10.1016/j.ndteint.2003.10.005

[16] Loughlin, P. J. (n.d.). Time-frequency moments, " eyelets" and machine faults. 4391(2001), 44-50.

[17] Loughlin, P., Cakrak, F., \& Cohen, L. (2000). Conditional moments analysis of transients with application to helicopter fault data. Mechanical Systems and Signal Processing, 14(4), 511-522. https://doi.org/10.1006/mssp.1999.1287

[18] Loughlin, P. J., \& Davidson, K. L. (2000). Instantaneous spectral skew and kurtosis. IEEE Signal Processing Workshop on Statistical Signal and Array Processing, SSAP, 574-578. https://doi.org/10.1109/ssap.2000.870190

[19] Davidson, K. L., \& Loughlin, P. J. (2000). Instantaneous spectral moments. Journal of the Franklin Institute, 337(4), 421-436. https://doi.org/10.1016/s0016-0032(00)00034-x

[20] Fang, L., Wang, B., \& Zhai, M. (2019). On the spectral moment of quasi-bicyclic graphs. Applied Mathematics and Computation, 363, 0-6. https://doi.org/10.1016/j.amc.2019.124601

[21] Bakhteev, O. Y., \& Strijov, V. V. (2020). Comprehensive analysis of gradient-based hyperparameter optimization algorithms. Annals of Operations Research. https://doi.org/10.1007/s10479-019-03286-Z

[22] Hazan, E., Klivans, A., \& Yuan, Y. (2017). Hyperparameter optimization: A spectral approach. In arXiv.

[23] Feurer, M., \& Hutter, F. (2019). Hyperparameter Optimization. https://doi.org/10.1007/978-3-030-05318-5_1

[24] Chandrashekaran, A., \& Lane, I. R. (2017). Speeding up Hyperparameter Optimization by Extrapolation of Learning Curves Using Previous Builds. Lecture Notes in Computer Science (Including Subseries Lecture Notes in Artificial Intelligence and Lecture Notes in Bioinformatics). https://doi.org/10.1007/978-3-319-71249-9_29

[25] Falkner, S., Klein, A., \& Hutter, F. (2017). Combining Hyperband and Bayesian optimization. 7th Workshop on Bayesian Optimization at Neural Information Processing Systems (NIPS-BayesOpt).

[26] Li, L., Jamieson, K., DeSalvo, G., Rostamizadeh, A., \& Talwalkar, A. (2018). Hyperband: A novel bandit-based approach to hyperparameter optimization. Journal of Machine Learning Research.

[27] Rojas-Dominguez, A., Padierna, L. C., Carpio Valadez, J. M., PugaSoberanes, H. J., \& Fraire, H. J. (2017). Optimal Hyper-Parameter Tuning of SVM Classifiers with Application to Medical Diagnosis. IEEE Access. https://doi.org/10.1109/ACCESS.2017.2779794

[28] Czarnecki, W. M., Podlewska, S., \& Bojarski, A. J. (2015). Robust optimization of SVM hyperparameters in the classification of bioactive compounds. Journal of Cheminformatics. https://doi.org/10.1186/s13321015-0088-0

[29] Hutter, F., Kotthoff, L., \& Vanschoren, J. (2019). Automated machine learning : methods, systems, challenges. In Springer.

[30] Bergstra, J., Yamins, D., \& Cox, D. D. (2013). Making a science of model search: Hyperparameter optimization in hundreds of dimensions for vision architectures. 30th International Conference on Machine Learning, ICML 2013.

[31] Bergstra, J., \& Bengio, Y. (2012). Random search for hyper-parameter optimization. Journal of Machine Learning Research

[32] Hyperparameter Optimization in Classification Learner AppMATLAB \& Simulink, Mathworks.com, 2021. [Online]. Available: https://www.mathworks.com/help/stats/hyperparameter-optimizationin-classification-learner-app.html\#mw_10fc128a-9d9b-4531-9c1df945fd9cfcd2. [Accessed: 02- Jan- 2021]. 


\section{APPENDIX}

TABLE I

CONFUSION MATRIX OF MOMENTS FEATURES USING J48 ALGORITHM

\begin{tabular}{|c|c|c|c|}
\hline Healthy & Chipped & Missing & Classified as \\
\hline 13 & 0 & 2 & Healthy \\
\hline 1 & 12 & 2 & Chipped \\
\hline 0 & 0 & 15 & Missing \\
\hline
\end{tabular}
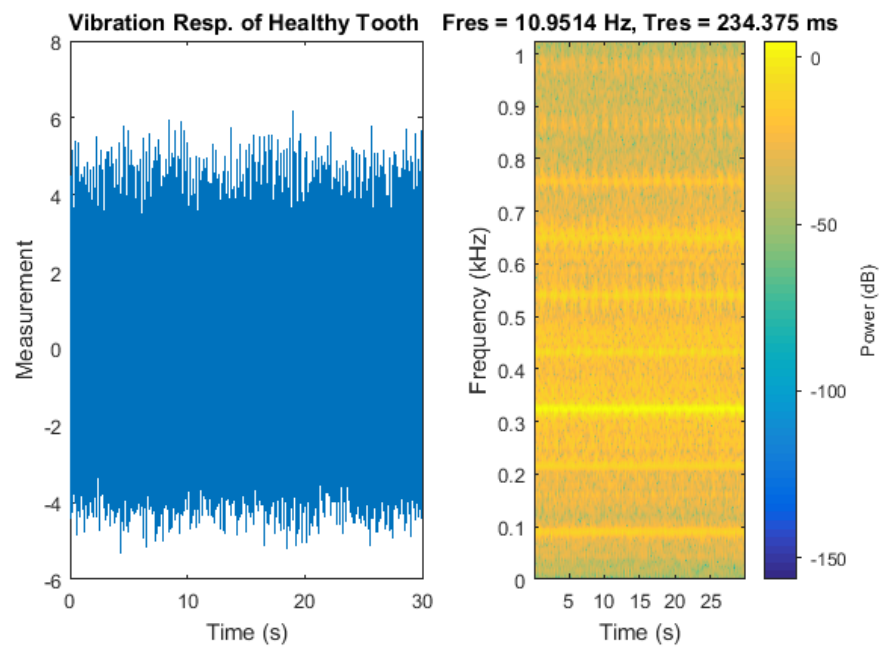

Fig. 13. Spectrogram of healthy tooth condition.
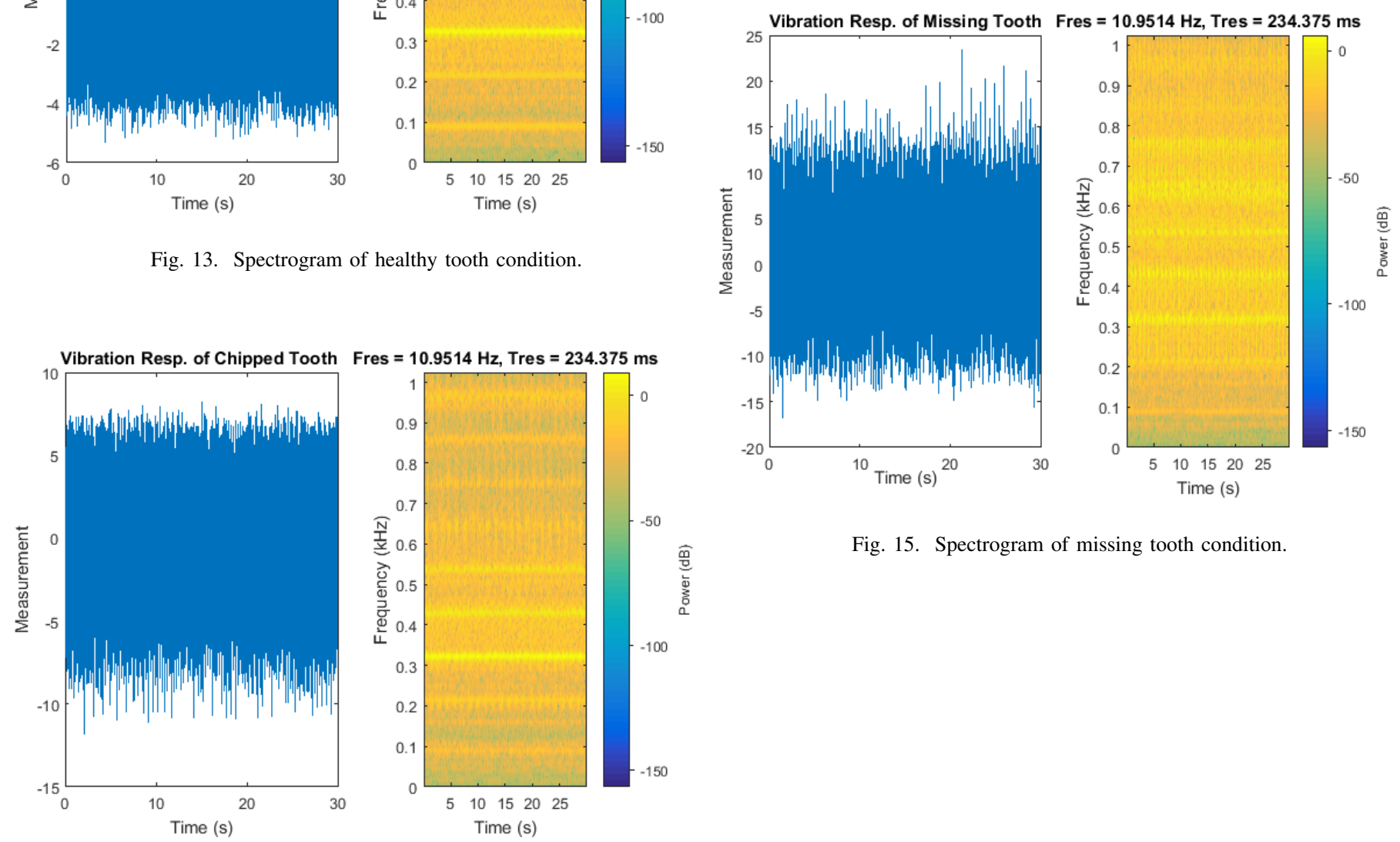

Fig. 15. Spectrogram of missing tooth condition.

Fig. 14. Spectrogram of chipped tooth condition. 